\title{
PRMT1 and PRMT4 Regulate Oxidative Stress-Induced Retinal Pigment Epithelial Cell Damage in SIRT1-Dependent and SIRT1-Independent Manners
}

\author{
Dong-Il Kim, ${ }^{1,2}$ Min-Jung Park, ${ }^{1,3}$ Joo-Hee Choi, ${ }^{1}$ In-Seon Kim, ${ }^{4}$ Ho-Jae Han, ${ }^{5}$ \\ Kyung-Chul Yoon, ${ }^{6}$ Sang-Woo Park, ${ }^{6}$ Min-Young Lee, ${ }^{7}$ Ki-Seok Oh, ${ }^{1}$ and Soo-Hyun Park ${ }^{1}$ \\ ${ }^{1}$ College of Veterinary Medicine, Chonnam National University, Gwangju 500-757, Republic of Korea \\ ${ }^{2}$ Life Science Institute, University of Michigan, Ann Arbor, MI 48109, USA \\ ${ }^{3}$ Departments of Molecular \& Integrative Physiology and Medicine, University of Michigan, Ann Arbor, MI 48109, USA \\ ${ }^{4}$ Division of Applied Bioscience and Biotechnology, Institute of Environmentally-Friendly Agriculture, \\ College of Agriculture and Life Sciences, Chonnam National University, Gwangju 500-757, Republic of Korea \\ ${ }^{5}$ College of Veterinary Medicine and Research Institute for Veterinary Science and BK21 PLUS Creative Veterinary Research Center, \\ Seoul National University, Seoul 151-741, Republic of Korea \\ ${ }^{6}$ Department of Ophthalmology, Chonnam National University Medical School and Hospital, Gwangju 501-757, Republic of Korea \\ ${ }^{7}$ Department of Molecular Physiology, College of Pharmacy, Kyungpook National University, Taegu 702-701, Republic of Korea
}

Correspondence should be addressed to Ki-Seok Oh; gsoh@jnu.ac.kr and Soo-Hyun Park; parksh@chonnam.ac.kr

Received 3 April 2015; Accepted 3 June 2015

Academic Editor: Mark A. Yorek

Copyright (C) 2015 Dong-Il Kim et al. This is an open access article distributed under the Creative Commons Attribution License, which permits unrestricted use, distribution, and reproduction in any medium, provided the original work is properly cited.

\begin{abstract}
Oxidative stress-induced retinal pigment epithelial (RPE) cell damage is involved in the progression of diabetic retinopathy. Arginine methylation catalyzed by protein arginine methyltransferases (PRMTs) has emerged as an important histone modification involved in diverse diseases. Sirtuin (SIRT1) is a protein deacetylase implicated in the onset of metabolic diseases. Therefore, we examined the roles of type I PRMTs and their relationship with SIRT1 in human RPE cells under $\mathrm{H}_{2} \mathrm{O}_{2}$-induced oxidative stress. $\mathrm{H}_{2} \mathrm{O}_{2}$ treatment increased PRMT1 and PRMT4 expression but decreased SIRT1 expression. Similar to $\mathrm{H}_{2} \mathrm{O}_{2}$ treatment, PRMT1 or PRMT4 overexpression increased RPE cell damage. Moreover, the $\mathrm{H}_{2} \mathrm{O}_{2}$-induced RPE cell damage was attenuated by PRMT1 or PRMT4 knockdown and SIRT1 overexpression. In this study, we revealed that SIRT1 expression was regulated by PRMT1 but not by PRMT4. Finally, we found that PRMT1 and PRMT4 expression is increased in the RPE layer of streptozotocin-treated rats. Taken together, we demonstrated that oxidative stress induces apoptosis both via PRMT1 in a SIRT1-dependent manner and via PRMT4 in a SIRT1-independent manner. The inhibition of the expression of type I PRMTs, especially PRMT1 and PRMT4, and increased SIRT1 could be therapeutic approaches for diabetic retinopathy.
\end{abstract}

\section{Introduction}

Diabetic retinopathy is the leading cause of blindness. The breakdown of the blood-retinal barrier (BRB) mediated by oxidative stress is related to the progression of diabetic retinopathy $[1,2]$. Retinal pigment epithelial (RPE) cells are a vital component of the outer BRB and are vulnerable to oxidative stress [3]. However, the molecular mechanisms of oxidative stress-induced RPE cell damage are not fully understood.
Protein arginine methyltransferases (PRMTs) catalyse the methylation of the arginine residues of histone and nonhistone proteins. Mammals possess nine PRMTs, which are divided into three types according to their method of methylation. Type 1 PRMTs (PRMT1, PRMT2, PRMT3, PRMT4, PRMT6, and PRMT8) catalyse asymmetric dimethylation at arginine residues, whereas type II PRMTs (PRMT5 and PRMT9) catalyse symmetric dimethylation, and type III PRMTs (PRMT7) catalyse monomethylation [4]. PRMT1 is 
thought to be involved in diabetic retinopathy, as PRMT1 expression is increased via the generation of reactive oxygen species (ROS) in the retinas of streptozotocin-treated rats and high-glucose-treated bovine retinal capillary endothelial cells, which are a crucial component of the inner BRB [5]. However, the regulation of PRMTs by oxidative stress in RPE cells has not been elucidated.

Sirtuin (SIRT1), a mammalian ortholog of yeast Sir2 (Silent Information Regulator 2), is an NAD-dependent histone deacetylase that regulates diverse physiological and pathophysiological processes, such as senescence, circadian rhythms, autophagy, and apoptosis [6]. In RPE cells, decreased SIRT1 expression caused by ultraviolet light is related to RPE cell damage [7]. The treatment of RPE cells with resveratrol, which increases SIRT1 activity, suppresses inflammatory cytokine-induced vascular endothelial growth factor (VEGF) secretion, which is involved in age-related macular degeneration (AMD) [8]. These reports suggest that SIRT1 protects against RPE cell dysregulation. However, the mechanisms regulating SIRT1 in RPE cells have not been evaluated.

In this study, we evaluated type I PRMT expression and SIRT1 expression under hydrogen peroxide- $\left(\mathrm{H}_{2} \mathrm{O}_{2}^{-}\right)$ induced oxidative stress and demonstrated that oxidative stress-induced PRMT1 expression increases RPE cell apoptosis via SIRT1 downregulation, whereas PRMT4 does so independently of SIRT1 expression.

\section{Materials and Methods}

2.1. Materials. Dulbecco's Modified Eagle's Medium (DMEM), Ham's nutrient mixture F-12, and fetal bovine serum (FBS) were purchased from Life Technologies (Gibco BRL, Grand Island, NY, USA). Hydrogen peroxide was obtained from Sigma-Aldrich (St. Louis, MO, USA). PRMT1 antibody (\#2449), PRMT4 antibody (\#4438), PARP1 antibody (\#9532), and Caspase-3 antibody (\#9662) were purchased from Cell Signaling Technology (Beverly, MA, USA). SIRT1 antibody (sc-15404) and $\beta$-actin antibody (sc-1616) were purchased from Santa Cruz Biotechnology (CA, USA). HA antibody (MMS-101R) was obtained from Covance (WI, USA). PRMT3 antibody was kindly provided by Mark T. Bedford (University of Texas, M. D. Anderson Cancer Center, Smithville, TX). All reagents were of the highest purity commercially available.

2.2. Cell Culture. The human RPE cell line ARPE-19 was obtained from the American Type Culture Collection (ATCC, Rockville, MD, USA). ARPE-19 cells were grown in DMEM/Ham's F-12 (1:1) supplemented with 10\% fetal bovine serum (FBS) at $37^{\circ} \mathrm{C}$ in $5 \% \mathrm{CO}_{2}$ in air. Stock cultures of ARPE19 cells were subcultured once a week (split ratio 1:6). Cells were grown to confluence in $60 \mathrm{~mm}$ dishes in DMEM/Ham's F-12 with $15 \mathrm{mM}$ HEPES buffer, 10\% FBS, $5.5 \mathrm{mM}$ glucose, $0.35 \%$ additional sodium bicarbonate, $2.5 \mathrm{mM}$ L-glutamine, and $1 \%$ penicillin/streptomycin at $37^{\circ} \mathrm{C}$. The media were changed every other day. Passaged cells were plated to yield near-confluent cultures at the end of the experiments.
2.3. MTT Assay. ARPE-19 cells were cultured on a 96-well plate in DMEM/Ham's F-12 (1:1) medium supplemented with $10 \%$ FBS. After treatments, the cells were treated with $500 \mu \mathrm{g} / \mathrm{mL}$ of 3-(4,5-dimethysssl-thiazol-2-yl)-2,5-diphenyl tetrazolium bromide (MTT; Sigma) and incubated for $3 \mathrm{~h}$ in a $\mathrm{CO}_{2}$ incubator. Cells with a functional mitochondrial succinate dehydrogenase can convert MTT to formazan. The formazan crystals formed were solubilized in DMSO (Sigma) and measured with an ELx808 microplate spectrophotometer reader at $\lambda=570 \mathrm{~nm}$ (BioTek).

2.4. Western Blotting. Western blot analysis was performed according to methods described previously [9]. Transferred membranes were probed with various antibodies. The bands were visualized with Luminescent image analyzer (ImageQuant LAS 4000, GE Healthcare, UK) using Amersham ECL Western Blotting Detection Reagents (GE Healthcare, UK).

2.5. Plasmids and DNA Transfection. The Flag, Flag-SIRT1, and Flag-SIRT1 H363Y plasmids were kindly provided by Dr. Hueng-Sik Choi (School of Biological Sciences and Technology, Chonnam National University, Korea). HA, HA-PRMT1, and HA-PRMT4 were kindly provided by Dr. Fukamizu A (Life Science Center of Tsukuba Advanced Research Alliance, University of Tsukuba, Japan). The plasmids were transfected into ARPE-19 cells using PolyExpress transfection reagent (Excellgen, Gaithersburg, MD, USA) in accordance with the manufacturer's instructions.

2.6. siRNA Transfection. siRNA for PRMT1 (sc-41069; Santa Cruz Biotechnology, Santa Cruz, CA, USA), PRMT4 (sc44875; Santa Cruz Biotechnology), and scramble siRNA (Qiagen, Hilden, Germany) were used to silence endogenous PRMT1 and PRMT4 expression. Lipofectamine RNAiMAX reagent (Invitrogen, Carlsbad, CA, USA) was used to transfect each siRNA $(30 \mathrm{nM})$ following reverse transfection in accordance with the manufacturer's instructions.

2.7. Animal Experiments. Hyperglycemia was induced in overnight fasted, 10 -week-old male SD rats $(n=7)$ by intraperitoneal injection of streptozotocin $(55 \mathrm{mg} / \mathrm{kg})$ dissolved in cold and fresh citrate buffer (0.1 M and $\mathrm{pH} 4.5)$. Control rats $(n=7)$ were injected with citrate buffer. Three days after STZ injection, plasma glucose level was determined after overnight fasting with Accu-Chek Aviva (Roche, Swiss). Rats with a blood glucose level of $300 \mathrm{mg} / \mathrm{dL}$ or higher were considered as diabetes. After 2 weeks, for preparation of cryosections, the rats were anaesthetized and eyeballs were enucleated, and then they were killed by $\mathrm{CO}_{2}$ inhalation. All animal experiments were performed in accordance with National Institutes of Health animal research standards. And protocols were approved by the Chonnam National University Laboratory Animal Research Center.

2.8. Immunohistochemistry (IHC) and Digital Image Analysis. Eyeballs were fixed in $4 \%$ paraformaldehyde in phosphatebuffered saline (PBS, pH 7.4) for 2 hours at $4^{\circ} \mathrm{C}$. Eyeballs were 


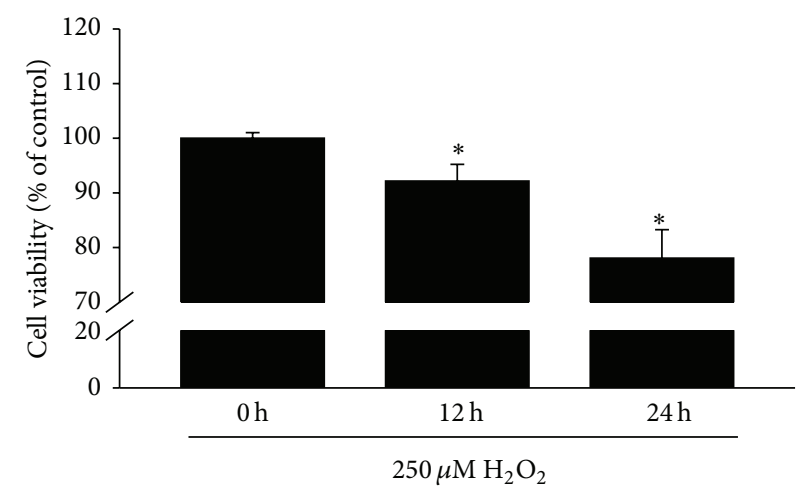

(a)
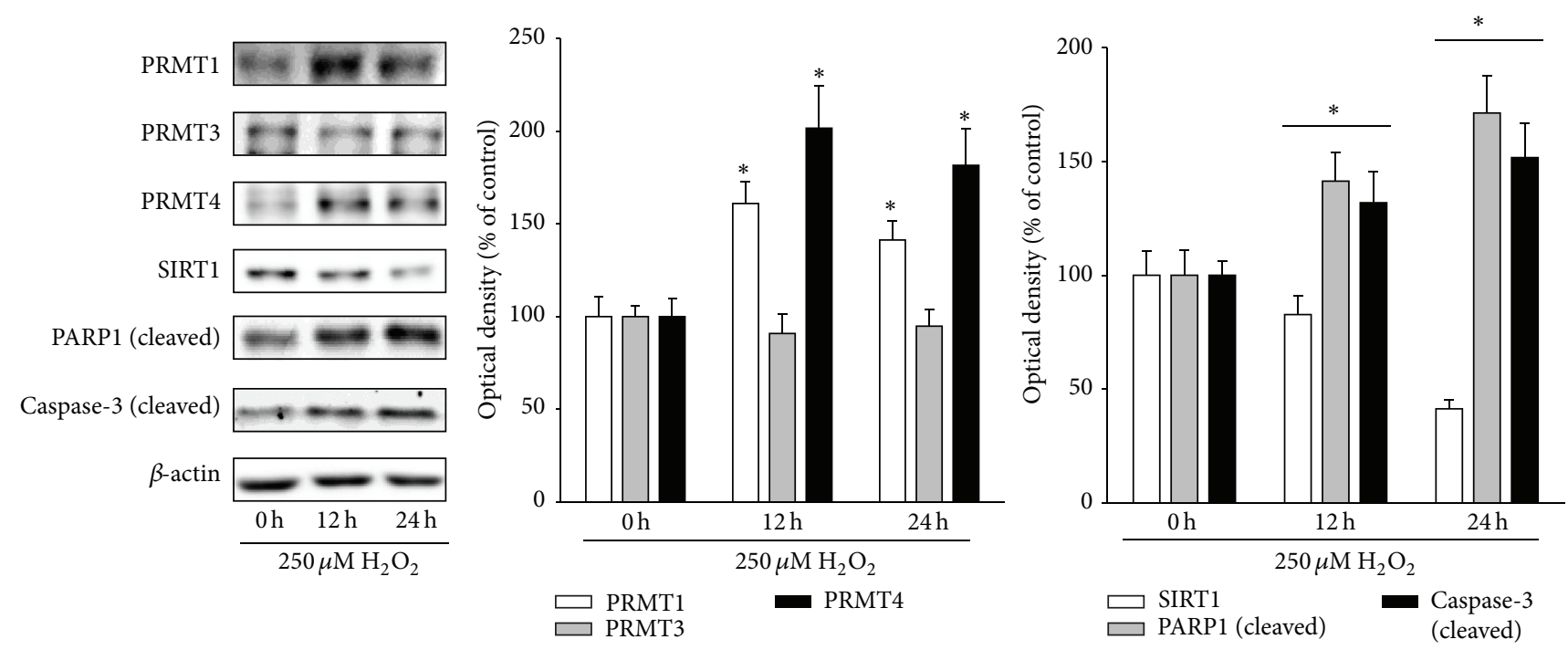

(b)

FIGURE 1: $\mathrm{H}_{2} \mathrm{O}_{2}$ increases PRMT1 and PRMT4 expression but decreases SIRT1 expression. (a, b) ARPE-19 cells were treated with $250 \mu \mathrm{M} \mathrm{H}_{2} \mathrm{O}_{2}$ for 12 and $24 \mathrm{~h}$. (a) Cell viability was measured by the MTT assay. The data represent the means \pm SEM of three independent experiments, each performed in triplicate. ${ }^{*} P<0.05$ versus $0 \mathrm{~h}$. (b) Cell extracts were subjected to Western blotting with the indicated antibodies. The data represent the means \pm SEM of three independent experiments. ${ }^{*} \mathrm{P}<0.05$ versus $0 \mathrm{~h}$.

frozen in OCT compound (Cellpath, Hemel Hempstead, UK). Cryosections of the retina $(10 \mu \mathrm{m})$ were cut through the optic nerve head. Fixation was performed with precooled $\left(-20^{\circ} \mathrm{C}\right)$ acetone for $10 \mathrm{~min}$. After allowing acetone to evaporate, immunostaining was performed according to the Vectastain ABC kit (Vector Labs; PK-6101). Briefly, endogenous peroxidase activity was quenched by $0.3 \% \mathrm{H}_{2} \mathrm{O}_{2}$ treatment for $30 \mathrm{~min}$ and then sections were blocked with goat serum for $20 \mathrm{~min}$ and then probed with PRMT1 antibody (diluted $1: 200$ ) or PRMT4 antibody (diluted 1:300) for $60 \mathrm{~min}$. After washing with PBS, sections were incubated with biotinylated secondary antibody for $30 \mathrm{~min}$ and then incubated with $\mathrm{ABC}$ reagent for $30 \mathrm{~min}$. DAB (Vector Labs; SK-4100) was used for peroxidase substrate solution. Hematoxylin staining was performed for counter-staining. The immunohistochemistrystained sections were observed using a BX-40 apparatus (Olympus, Tokyo, Japan) with an eXcope X3 digital camera (DIXI Optics, Daejeon, South Korea). Semiautomated analysis protocol was used to quantify the IHC images.
Using imageJ, pure DAB images were deconvoluted from IHC images. The pixel intensities of pure DAB images were analyzed with histogram range from 0 to 255 . The lower pixel value represents the higher positive signals of $\mathrm{DAB}$. The pixel values were mainly spread from 111 to 200 . The values between 111 and 140 were considered as high positive, 141-170 were positive, and 171-200 were low positive.

2.9. Statistical Analysis. The results were expressed as the mean \pm SEM. Values are the mean \pm SEM of three or four independent experiments. All the experiments were analyzed by analysis of variance (ANOVA). A $P$ value $<0.05$ was considered significant.

\section{Results}

3.1. $\mathrm{H}_{2} \mathrm{O}_{2}$ Increases PRMT1 and PRMT4 Expression and Decreases SIRT1 Expression. To induce oxidative stress, 


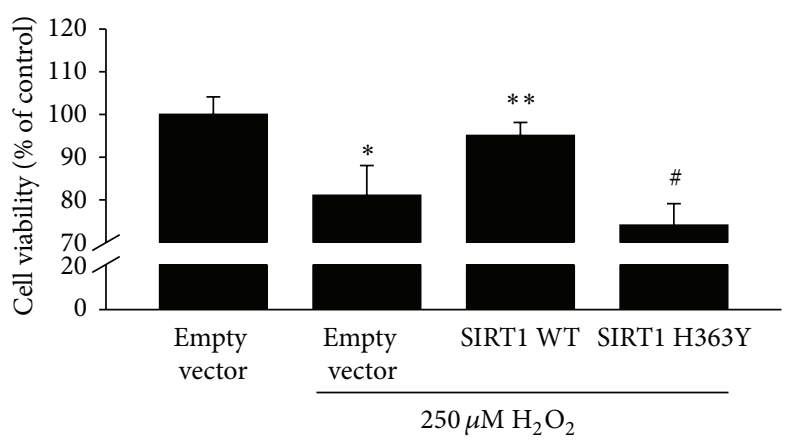

(a)
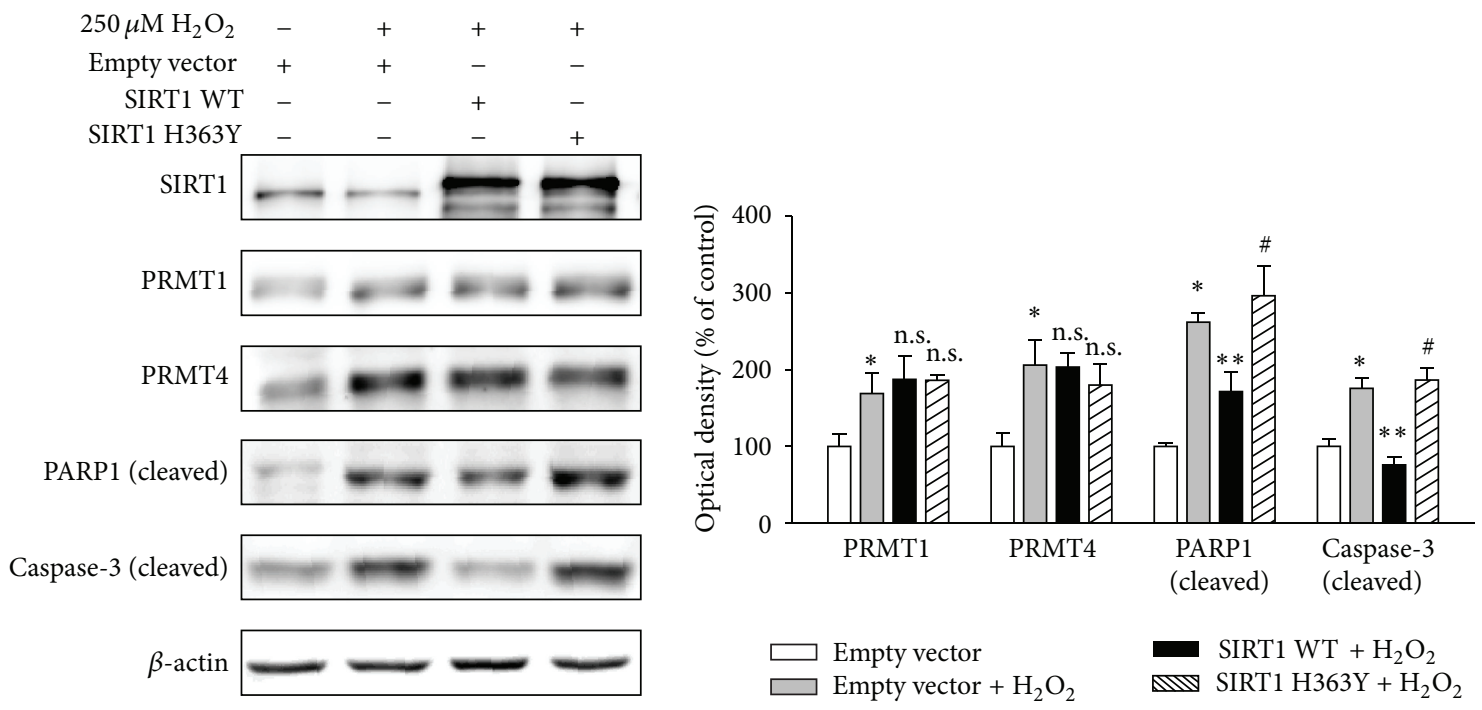

(b)

FIGURE 2: SIRT1 overexpression attenuates oxidative stress-induced RPE cell apoptosis. (a, b) ARPE-19 cells were transfected with empty vector, SIRT1 WT, or SIRT1 H363Y plasmids. After $24 \mathrm{~h}$, the medium was changed to serum-free medium and $250 \mu \mathrm{M} \mathrm{H}_{2} \mathrm{O}_{2}$ was added for $24 \mathrm{~h}$. (a) Cell viability was measured by the MTT assay. The data represent the means \pm SEM of three independent experiments, each performed in triplicate. ${ }^{*} P<0.05$ versus empty vector, ${ }^{* *} P<0.05$ versus empty vector $+250 \mu \mathrm{M} \mathrm{H}_{2} \mathrm{O}_{2}$, and ${ }^{*} P<0.05$ versus SIRT1 WT $+250 \mu \mathrm{M} \mathrm{H}_{2} \mathrm{O}_{2}$. (b) Cell extracts were subjected to Western blotting with the indicated antibodies. The data represent the means \pm SEM of three independent experiments. ${ }^{*} P<0.05$ versus empty vector, ${ }^{* *} P<0.05$ versus empty vector $+250 \mu \mathrm{M} \mathrm{H}_{2} \mathrm{O}_{2}$, and ${ }^{\#} P<0.05$ versus SIRT1 WT $+250 \mu \mathrm{M} \mathrm{H}_{2} \mathrm{O}_{2}$ (n.s. = nonspecific).

human retinal pigment epithelial cells (ARPE-19 cells) were treated with $250 \mu \mathrm{M} \mathrm{H} \mathrm{H}_{2} \mathrm{O}_{2}$. As expected, $\mathrm{H}_{2} \mathrm{O}_{2}$ treatment decreased cell viability and increased the cleavage of PARP1 and caspase-3, which are associated with RPE cell apoptosis (Figures 1(a) and 1(b)) [10]. Furthermore, $\mathrm{H}_{2} \mathrm{O}_{2}$ treatment increased PRMT1 and PRMT4 expression and decreased SIRT1 expression, while PRMT3 expression was unchanged (Figure 1(b)).

3.2. SIRT1 Overexpression Attenuates Oxidative Stress-Induced RPE Cell Apoptosis. As $\mathrm{H}_{2} \mathrm{O}_{2}$ treatment decreased SIRT1 expression, we postulated that oxidative stress-induced RPE cell damage is regulated by SIRT1. To confirm this, ARPE-19 cells were transfected with empty vector or SIRT1 followed by $\mathrm{H}_{2} \mathrm{O}_{2}$. SIRT1 overexpression restored the cell viability lowered by $\mathrm{H}_{2} \mathrm{O}_{2}$ treatment (Figure 2(a)). Moreover, the increased cleavage of PARP1 and caspase- 3 was attenuated by
SIRT1 overexpression (Figure 2(b)). However, transfection of SIRT1 H363Y, an enzymatic-dead mutant that lacks deacetylase activity because histidine 363 is converted to tyrosine [11], did not restore cell viability or attenuate PARP1 and caspase-3 cleavage (Figures 2(a) and 2(b)). SIRT1 or SIRT1 H363Y overexpression did not influence PRMT1 or PRMT4 expression under oxidative stress (Figure 2(b)).

3.3. PRMT1 or PRMT4 Overexpression Increases RPE Cell Apoptosis and PRMT1 Overexpression Decreases SIRT1 Expression. $\mathrm{H}_{2} \mathrm{O}_{2}$ treatment increased PRMT1 and PRMT4 expression (Figure 1(b)). To determine the effect of the increased PRMT1 or PRMT4 on RPE cell apoptosis, ARPE-19 cells were transfected with HA, HA-PRMT1, or HA-PRMT4. PRMT1 overexpression decreased cell viability and SIRT1 expression (Figures 3(a) and 3(b)). Moreover, PRMT1 overexpression increased the cleavage of PARP1 and caspase-3 (Figure 3(b)). 


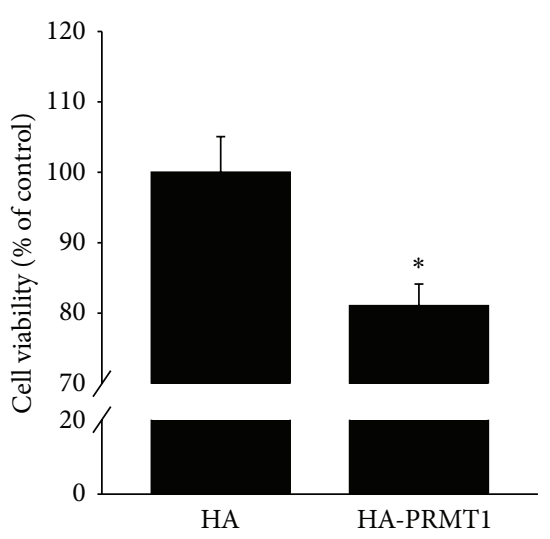

(a)

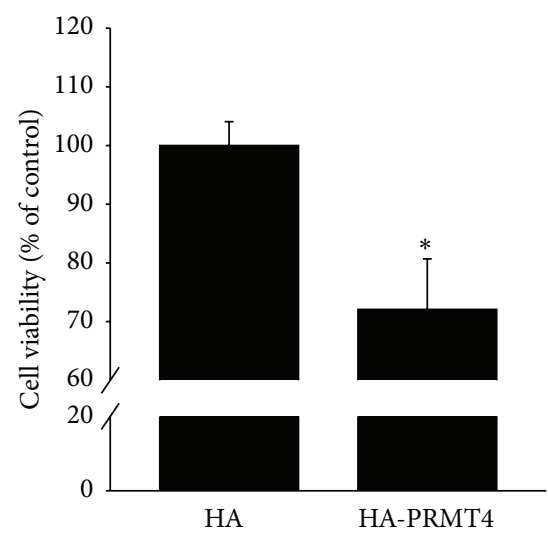

(c)

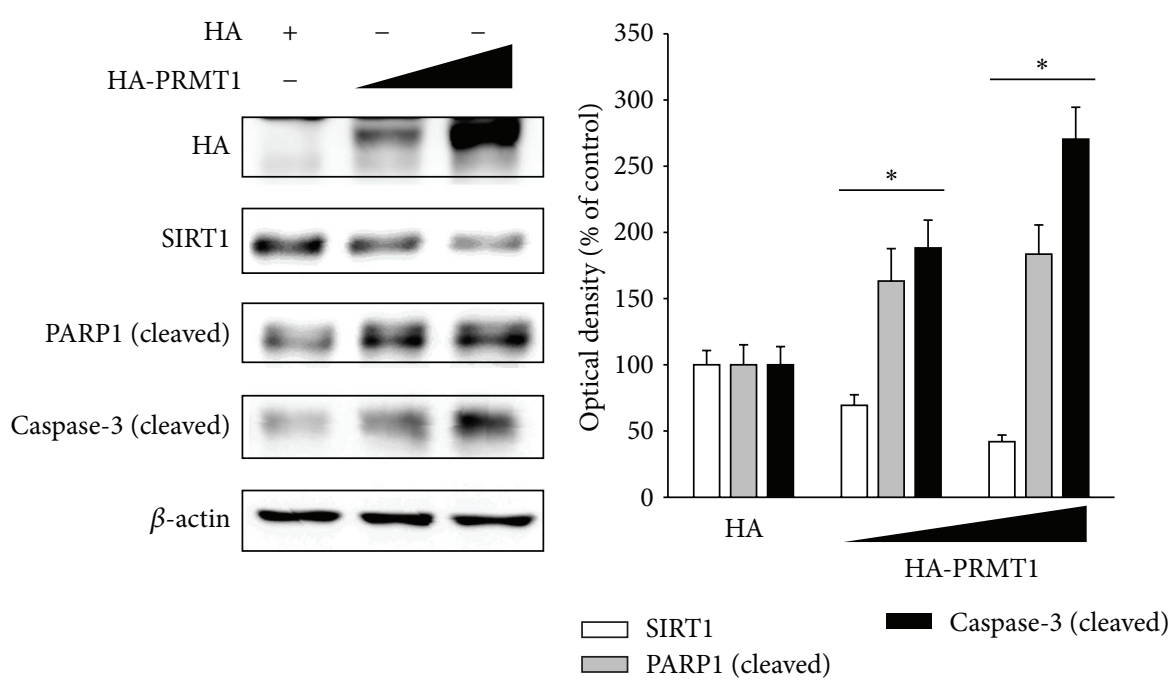

(b)
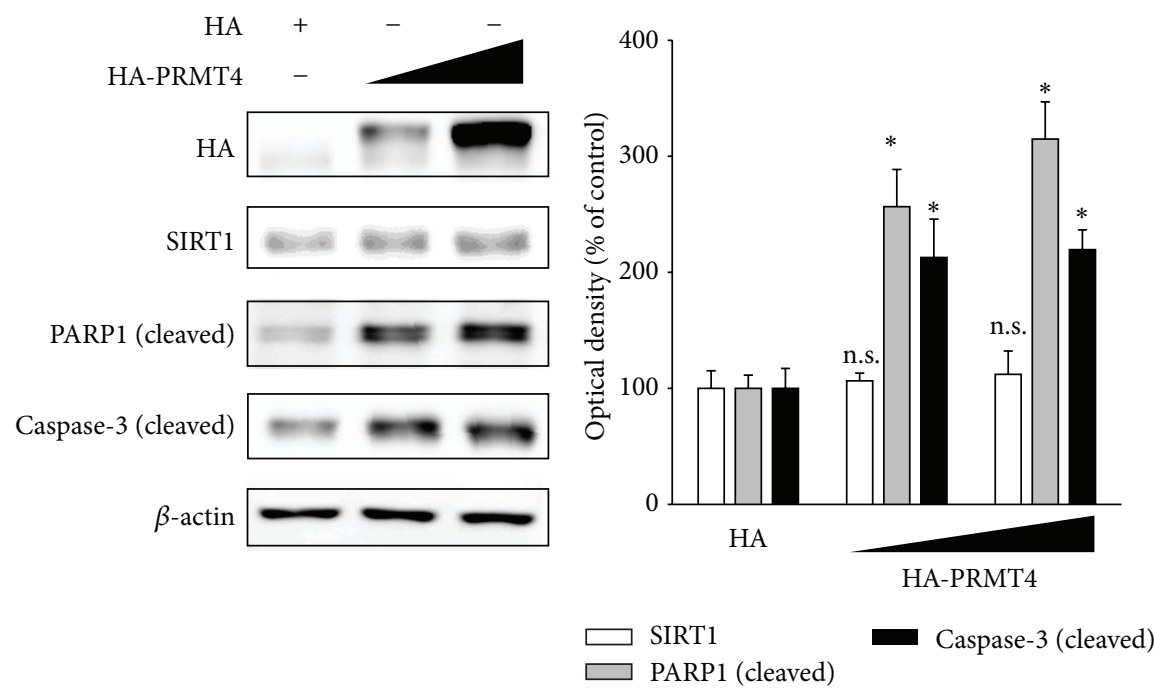

(d)

FIgURE 3: PRMT1 or PRMT4 overexpression increases RPE cell apoptosis, while PRMT1 overexpression decreases SIRT1 expression. (a, b) ARPE-19 cells were transfected with HA or HA-PRMT1 plasmid DNA. After $36 \mathrm{~h}$, (a) cell viability was measured by the MTT assay. The data represent the means \pm SEM of three independent experiments, each performed in triplicate. ${ }^{*} P<0.05$ versus HA. (b) Cell extracts were subjected to Western blotting with the indicated antibodies. The data represent the means \pm SEM of three independent experiments. ${ }^{*} P<0.05$ versus HA. (c, d) ARPE-19 cells were transfected with HA or HA-PRMT4 plasmid DNA. After 36 h, (c) cell viability was measured by the MTT assay. The data represent the means \pm SEM of three independent experiments, each performed in triplicate. ${ }^{*} P<0.05$ versus $H A$. (d) Cell extracts were subjected to Western blotting with the indicated antibodies. The data represent the means \pm SEM of three independent experiments. ${ }^{*} P<0.05$ versus HA (n.s. $=$ nonspecific).

PRMT4 overexpression also decreased cell viability and increased the cleavage of PARP1 and caspase-3 but did not alter SIRT1 expression (Figures 3(c) and 3(d)).

\subsection{PRMT1 or PRMT4 Knockdown Attenuates Oxidative} Stress-Induced RPE Cell Damage and PRMT1 Expression Regulates SIRT1 Expression. To confirm these findings, PRMT1 or PRMT4 expression was silenced by siRNA transfection (Figure 4(a)). PRMT1 or PRMT4 knockdown restored the $\mathrm{H}_{2} \mathrm{O}_{2}$-induced decrease in cell viability (Figure 4(b)). Moreover, the increased cleavage of PARP1 and caspase-3 was attenuated by knockdown of PRMT1 or PRMT4 (Figure 4(c)). The $\mathrm{H}_{2} \mathrm{O}_{2}$-induced SIRT1 downregulation was restored by PRMT1 knockdown but not by PRMT4 knockdown (Figure 4(c)).

3.5. PRMT1 and PRMT4 Expression Is Increased in the RPE Layer of Streptozotocin-Treated Rats. To confirm the increase of PRMT1 and PRMT4 expression in vivo, we generated rats with streptozotocin- (STZ-) induced diabetes, which show severe hyperglycemia and are known to have induced diabetic retinopathy via oxidative stress $[12,13]$. As shown in 


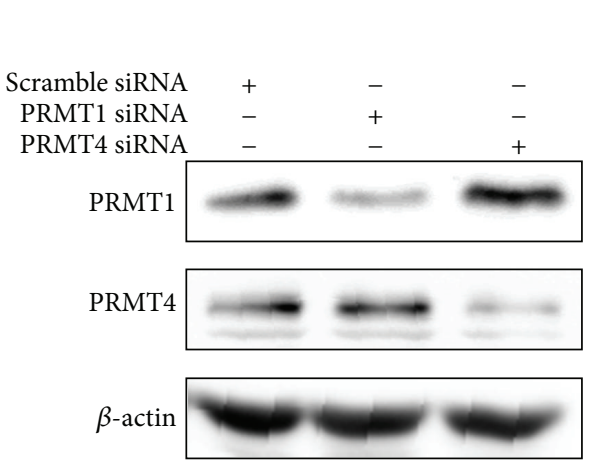

(a)

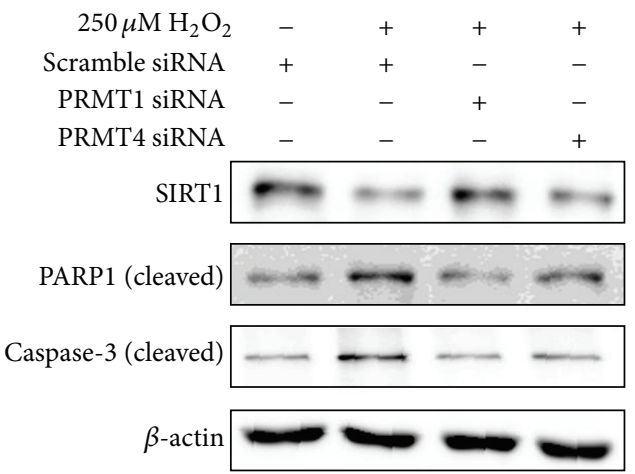

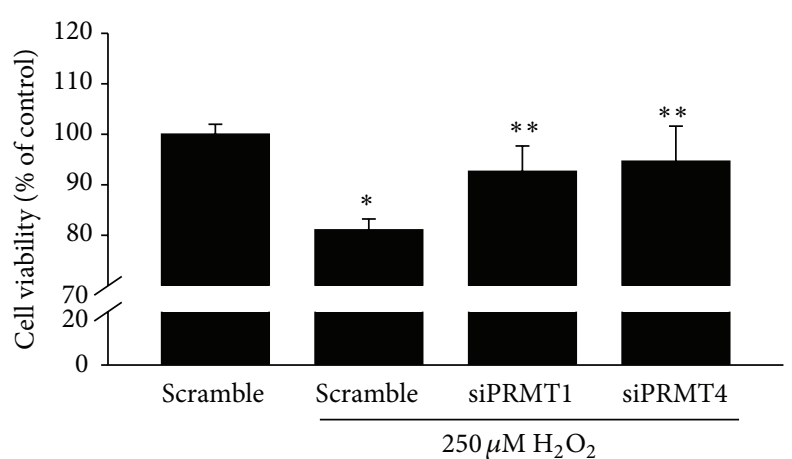

(b)

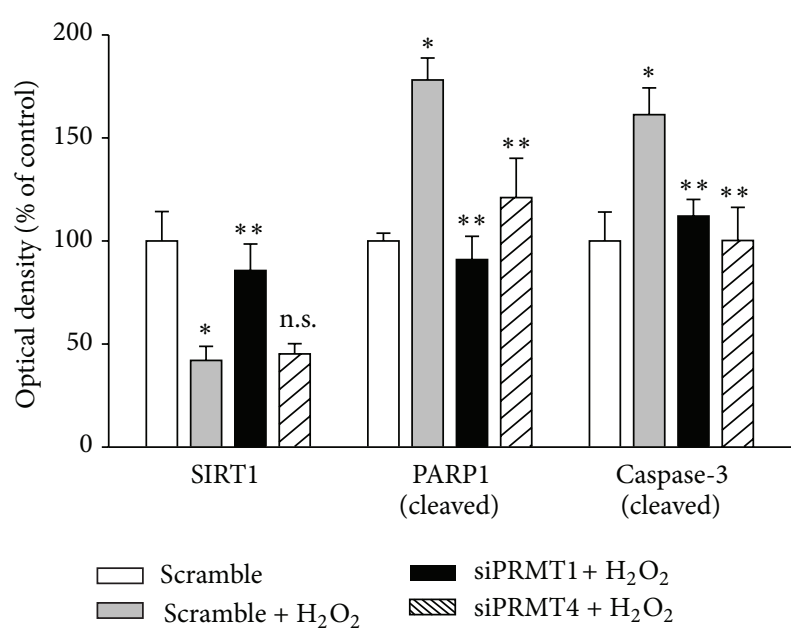

(c)

FIGURE 4: The knockdown of PRMT1 or PRMT4 attenuates oxidative stress-induced RPE cell damage and PRMT1 expression regulates SIRT1 expression. (a) ARPE-19 cells were transfected with scramble, PRMT1, or PRMT4 siRNA according to the reverse transfection method. After $36 \mathrm{~h}$, cell extracts were subjected to Western blotting with the indicated antibodies. (b, c) ARPE-19 cells were transfected with scramble, PRMT1, or PRMT4 siRNA according to the reverse transfection method. After $24 \mathrm{~h}$, the medium was changed to serum-free medium and $250 \mu \mathrm{M} \mathrm{H}_{2} \mathrm{O}_{2}$ was added for $24 \mathrm{~h}$. (b) Cell viability was measured by the MTT assay. The data represent the means \pm SEM of three independent experiments, each performed in triplicate. ${ }^{*} \mathrm{P}<0.05$ versus scramble siRNA, ${ }^{* *} \mathrm{P}<0.05$ versus scramble siRNA $+250 \mu \mathrm{M} \mathrm{H}_{2} \mathrm{O}_{2}$. (c) Cell extracts were subjected to Western blotting with the indicated antibodies. The data represent the means \pm SEM of three independent experiments. ${ }^{*} P<0.05$ versus scramble siRNA, ${ }^{* *} P<0.05$ versus scramble siRNA $+250 \mu \mathrm{M} \mathrm{H}_{2} \mathrm{O}_{2}$ (n.s. = nonspecific).

Figures 5(a) and 5(b), PRMT1 and PRMT4 expression was significantly increased in the RPE layer of STZ rats compared with vehicle-treated rats (white arrows). Furthermore, high positive and positive signals of PRMT1 and high positive signals of PRMT4 were greatly increased in STZ rats compared with vehicle-treated rats (Figures 5(a) and 5(b)).

\section{Discussion}

Type I PRMTs are important pathophysiological regulators as they promote the production of asymmetric dimethylarginine (ADMA), a metabolic by-product that inhibits nitric oxide synthase (NOS), which is involved in cardiovascular disease, diabetes, and other metabolic disorders [14-16]. In addition to producing ADMA, type I PRMTs regulate various cellular processes, such as transcription, RNA splicing, and signal transduction, by catalysing the asymmetric dimethylation of histone or nonhistone proteins [17]. Recent studies have revealed the role of type I PRMTs in diabetic nephropathy $[18,19]$. However, their roles in diabetic retinopathy are rarely known. In this study, we demonstrated that increased expression of the type I PRMTs, PRMT1 and PRMT4, induced RPE cell damage under oxidative stress. This was supported by the following evidence: (1) $\mathrm{H}_{2} \mathrm{O}_{2}$ treatment increased not only RPE cell damage but also PRMT1 and PRMT4 expression; (2) PRMT1 or PRMT4 overexpression increased RPE cell damage; (3) PRMT1 or PRMT4 knockdown attenuated the $\mathrm{H}_{2} \mathrm{O}_{2}$-induced RPE cell damage; and (4) PRMT1 and PRMT4 expression was increased in the RPE layer of STZtreated rats.

Many studies have demonstrated that oxidative stressinduced cellular damage is mediated by increased type I PRMT expression. For example, $\mathrm{H}_{2} \mathrm{O}_{2}$-induced oxidative 


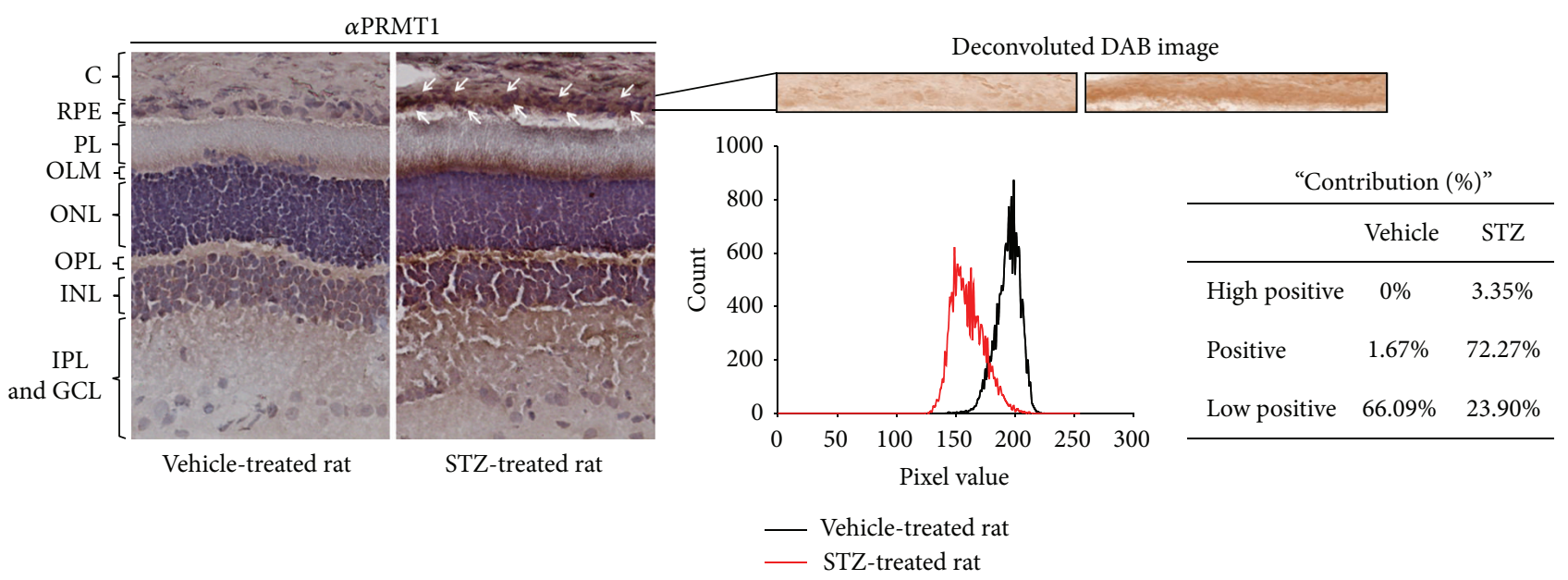

(a)

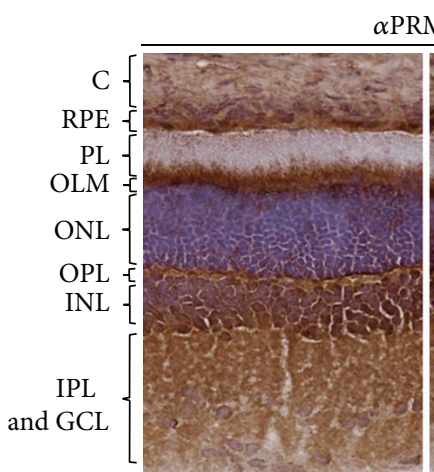

Vehicle-treated rat

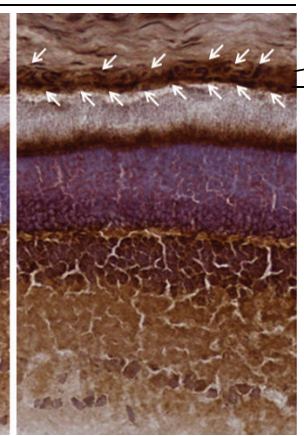

STZ-treated rat

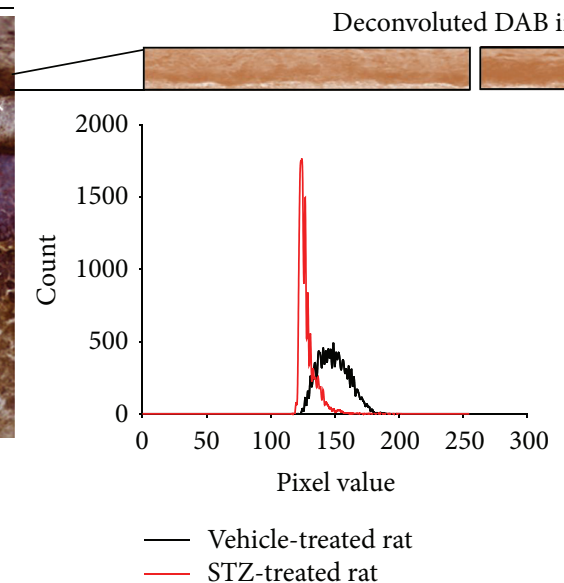

\begin{tabular}{lcc}
\multicolumn{3}{c}{ “Contribution (\%)" } \\
\hline & Vehicle & STZ \\
\hline High positive & $19.77 \%$ & $76.08 \%$ \\
Positive & $57.18 \%$ & $5.04 \%$ \\
Low positive & $4.26 \%$ & $0.08 \%$ \\
\hline
\end{tabular}

(b)

FIGURE 5: PRMT1 and PRMT4 expression is increased in the RPE layer of streptozotocin-treated rats. Eyeballs were enucleated from vehicle-treated and STZ-treated rats and cryosections were prepared. (a, b) PRMT1 (a) and PRMT4 (b) expressions were measured by immunohistochemistry analysis (C: choroid, RPE: retinal pigment epithelium, PL: photoreceptor layer, OLM: outer limiting membrane, ONL: outer nuclear layer, OPL: outer plexiform layer, INL: inner nuclear layer, IPL: inner plexiform layer, and GCL: ganglion-cell layer). Representative images were from at least three independent experiments. To quantify the DAB signaling, semiautomated analysis protocol was performed as described in Section 2.

stress increases PRMT3 expression, leading to increased ADMA generation in preglomerular vascular smooth muscle cells [20]. Treatment with human serum albumin, which induces oxidative stress in renal proximal tubular epithelial cells, increased PRMT1 expression [21]. In contrast, few studies have revealed the protective effects of type I PRMTs in oxidative stress. Very recently, Huang et al. reported that arsenic-induced oxidative stress recruits PRMT1 to the histone 4 arginine 3 and PRMT4 to the histone 3 arginine 17 for asymmetric dimethylation, which leads to increased ferritin transcription via the antioxidant responsive element in $\mathrm{HaCaT}$ cells (human keratinocytes) [22]. In the report by Huang et al., in contrast to our results, the expression of PRMT1 and PRMT4 was not changed and PRMT1 and PRMT4 increased ferritin to protect cells from oxidative stress. This discrepancy was likely due to the different cell types (retinal pigment epithelial cells versus keratinocyte) or the kind of oxidative stress $\left(\mathrm{H}_{2} \mathrm{O}_{2}\right.$ versus arsenic), as arsenic treatment induces superoxide anion $\left(\mathrm{O}_{2}{ }^{--}\right)$and hydroxyl radical $\left({ }^{\circ} \mathrm{OH}\right)$ production [23]. Moreover, PRMT1 and PRMT4 were examined within $6 \mathrm{~h}$ of arsenic treatment, while our experiment involved a relatively long duration of $\mathrm{H}_{2} \mathrm{O}_{2}$ treatment. Wang et al. reported that treatment with lithium and valproate acid, which protect against $\mathrm{H}_{2} \mathrm{O}_{2}$-induced oxidative stress, increase PRMT4 expression in NSC34 cells [24]. However, they did not establish the function of PRMT4.

In this study, we also showed that $\mathrm{H}_{2} \mathrm{O}_{2}$-induced SIRT1 downregulation is involved in RPE cell damage. Several lines of evidence support our findings. Wu et al. reported that SIRT1 overexpression reverses $\mathrm{H}_{2} \mathrm{O}_{2}$-mediated complement factor $\mathrm{H}$ downregulation in ARPE-19 cells [25]. Moreover, 


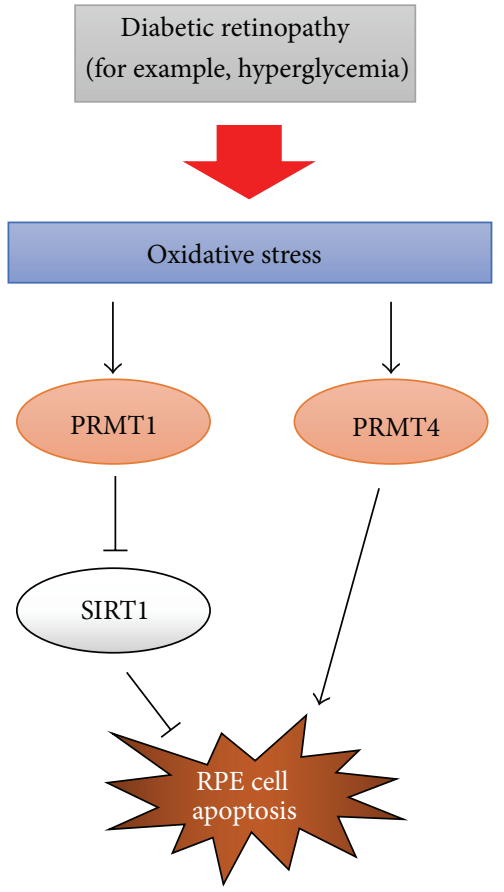

FIGURE 6: PRMT1 and PRMT4 regulate oxidative stress-induced RPE cell damage in SIRT1-dependent and SIRT1-independent manners.

Bhattacharya et al. reported that decreased SIRT1 expression in RPE cells induces p53 acetylation-mediated apoptosis, leading to the progression of age-related macular degeneration (AMD) [26]. Indeed, p53 and its target genes are closely involved in RPE cell apoptosis [27, 28]. As a deacetylase, SIRT1 inhibits p53 activity via deacetylation at lysine 382 [29]. In our study, transfection with an enzymatic-dead mutant, SIRT1, did not inhibit the $\mathrm{H}_{2} \mathrm{O}_{2}$-induced RPE cell apoptosis. Here, we provide novel evidence that SIRT1 expression and its enzymatic activity are vital for RPE cell maintenance.

Interestingly, we found that SIRT1 expression is negatively regulated by PRMT1 but not by PRMT4. Scalera et al. reported that red wine decreased PRMT1 expression in a SIRT1-dependent manner in human endothelial cells [30]. However, in our study, SIRT1 overexpression did not alter PRMT1 expression. This may be due to a cell-type-specific response (endothelial versus epithelial cells). We speculated that PRMT4 regulates SIRT1 expression, as PRMT4 increases the stability of SIRT1 mRNA by methylating HuR protein at arginine 217 in stem cells and HeLa cells [31, 32]. However, PRMT4 did not influence SIRT1 protein expression in ARPE19 cells. To our knowledge, this is the first report on the relationship between PRMT and SIRT1 in cell function. We provide the first evidence that PRMT1 regulates SIRT1 under oxidative stress.

Signaling induced by high-glucose is highly associated with oxidative stress [33]. Very recently, we reported that PRMT4 expression is increased by high-glucose in RPE cells [34]. Consistent with previous results, PRMT4 expression was increased in the RPE and outer limiting membrane
(OLM) layers of STZ rats. In addition, PRMT1 expression was increased in the RPE and overall layers of retina of STZ rats. It may be speculated that increased PRMT1 and PRMT4 expression in other layers by STZ treatment contributes to the progression of retinopathy. Further studies should be performed to reveal this speculation.

In this study, we demonstrated that oxidative stressinduced RPE cell damage is regulated by type I PRMTs (PRMT1 and PRMT4) and oxidative stress-induced SIRT1 downregulation is involved in the PRMT1-mediated RPE cell apoptosis pathway. Oxidative stress is a major cause of diabetic retinopathy. Taken together, our data suggest a model of signaling pathways involved in oxidative stress-induced RPE cell apoptosis (Figure 6). Therefore, the inhibition of type I PRMT expression, especially PRMT1 and PRMT4, and the increase in SIRT1 expression could be therapeutic approaches for diabetic retinopathy.

\section{Conflict of Interests}

The authors declare that there is no conflict of interests regarding the publication of this paper.

\section{Authors' Contribution}

Dong-Il Kim and Min-Jung Park contributed equally to this work. The two corresponding authors Ki-Seok Oh and SooHyun Park contributed equally to this work.

\section{Acknowledgments}

This work was supported financially by a research grant from the Korean Science \& Engineering Foundation (20100023627). This work was also supported by a grant from the Next-Generation BioGreen 21 Program (no. PJ009090), Rural Development Administration, Republic of Korea.

\section{References}

[1] J. Li, J. J. Wang, Q. Yu, K. Chen, K. Mahadev, and S. X. Zhang, "Inhibition of reactive oxygen species by lovastatin downregulates vascular endothelial growth factor expression and ameliorates blood-retinal barrier breakdown in $\mathrm{db} / \mathrm{db}$ mice: role of NADPH oxidase 4," Diabetes, vol. 59, no. 6, pp. 1528-1538, 2010.

[2] T. Frey and D. A. Antonetti, "Alterations to the blood-retinal barrier in diabetes: cytokines and reactive oxygen species," Antioxidants and Redox Signaling, vol. 15, no. 5, pp. 1271-1284, 2011.

[3] N. Strunnikova, C. Zhang, D. Teichberg et al., "Survival of retinal pigment epithelium after exposure to prolonged oxidative injury: a detailed gene expression and cellular analysis," Investigative Ophthalmology \& Visual Science, vol. 45, no. 10, pp. 3767-3777, 2004.

[4] M. T. Bedford and S. G. Clarke, "Protein arginine methylation in mammals: who, what, and why," Molecular Cell, vol. 33, no. 1, pp. 1-13, 2009.

[5] Y. Chen, X. Xu, M. Sheng, X. Zhang, Q. Gu, and Z. Zheng, "PRMT-1 and DDAHs-induced ADMA upregulation 
is involved in ROS- and RAS-mediated diabetic retinopathy," Experimental Eye Research, vol. 89, no. 6, pp. 1028-1034, 2009.

[6] T. Finkel, C.-X. Deng, and R. Mostoslavsky, "Recent progress in the biology and physiology of sirtuins," Nature, vol. 460, no. 7255, pp. 587-591, 2009.

[7] W.-W. Chou, K.-C. Chen, Y.-S. Wang, J.-Y. Wang, C.-L. Liang, and S.-H. H. Juo, "The role of SIRT1/AKT/ERK pathway in ultraviolet $\mathrm{B}$ induced damage on human retinal pigment epithelial cells," Toxicology in Vitro, vol. 27, no. 6, pp. 1728-1736, 2013.

[8] C. N. Nagineni, R. Raju, K. K. Nagineni et al., "Resveratrol suppresses expression of VEGF by human retinal pigment epithelial cells: potential nutraceutical for age-related macular degeneration," Aging and Disease, vol. 5, pp. 88-100, 2014.

[9] D. I. Kim and S. H. Park, "Sequential signaling cascade of IL6 and PGC- $1 \alpha$ is involved in high glucose-induced podocyte loss and growth arrest," Biochemical and Biophysical Research Communications, vol. 435, no. 4, pp. 702-707, 2013.

[10] S. K. Lim, M. J. Park, J. C. Lim et al., "Hyperglycemia induces apoptosis via $\mathrm{CB}_{1}$ activation through the decrease of FAAH 1 in retianl pigment epithelial cells," Journal of Cellular Physiology, vol. 227, no. 2, pp. 569-577, 2012.

[11] J. Luo, A. Y. Nikolaev, S.-I. Imai et al., "Negative control of p53 by Sir2alpha promotes cell survival under stress," Cell, vol. 107, no. 2, pp. 137-148, 2001.

[12] M. M. Anwar and A.-R. M. A. Meki, "Oxidative stress in streptozotocin-induced diabetic rats: effects of garlic oil and melatonin," Comparative Biochemistry and Physiology Part A: Molecular \& Integrative Physiology, vol. 135, no. 4, pp. 539-547, 2003.

[13] I. G. Obrosova, A. G. Minchenko, R. Vasupuram et al., "Aldose reductase inhibitor fidarestat prevents retinal oxidative stress and vascular endothelial growth factor overexpression in streptozotocin-diabetic rats," Diabetes, vol. 52, no. 3, pp. 864$871,2003$.

[14] H. Dayoub, V. Achan, S. Adimoolam et al., "Dimethylarginine dimethylaminohydrolase regulates nitric oxide synthesis: genetic and physiological evidence," Circulation, vol. 108, no. 24, pp. 3042-3047, 2003.

[15] F. Abbasi, T. Asagmi, J. P. Cooke et al., "Plasma concentrations of asymmetric dimethylarginine are increased in patients with type 2 diabetes mellitus," American Journal of Cardiology, vol. 88, no. 10, pp. 1201-1203, 2001.

[16] I. Palomo, A. Contreras, L. M. Alarcón et al., "Elevated concentration of asymmetric dimethylarginine (ADMA) in individuals with metabolic syndrome," Nitric Oxide, vol. 24, no. 4, pp. 224228, 2011.

[17] Y. Yang and M. T. Bedford, "Protein arginine methyltransferases and cancer," Nature Reviews Cancer, vol. 13, no. 1, pp. 37-50, 2013.

[18] A. Ojima, Y. Ishibashi, T. Matsui et al., "Glucagon-like peptide1 receptor agonist inhibits asymmetric dimethylarginine generation in the kidney of streptozotocin-induced diabetic rats by blocking advanced glycation end product-induced protein arginine methyltranferase-1 expression," American Journal of Pathology, vol. 182, no. 1, pp. 132-141, 2013.

[19] D. Kim, S. Lim, M. Park et al., "Ubiquitination-dependent CARM1 degradation facilitates Notchl-mediated podocyte apoptosis in diabetic nephropathy," Cellular Signalling, vol. 26, no. 9, pp. 1774-1782, 2014.

[20] Z. Luo, T. Teerlink, K. Griendling, S. Aslam, W. J. Welch, and C. S. Wilcox, "Angiotensin II and NADPH oxidase increase
ADMA in vascular smooth muscle cells," Hypertension, vol. 56, no. 3, pp. 498-504, 2010.

[21] Y. Kaida, S. Ueda, S.-I. Yamagishi et al., "Proteinuria elevates asymmetric dimethylarginine levels via protein arginine methyltransferase-1 overexpression in a rat model of nephrotic syndrome," Life Sciences, vol. 91, no. 9-10, pp. 301-305, 2012.

[22] B.-W. Huang, P. D. Ray, K. Iwasaki, and Y. Tsuji, “Transcriptional regulation of the human ferritin gene by coordinated regulation of Nrf2 and protein arginine methyltransferases PRMT1 and PRMT4," The FASEB Journal, vol. 27, no. 9, pp. 3763-3774, 2013.

[23] S. J. S. Flora, "Arsenic-induced oxidative stress and its reversibility," Free Radical Biology and Medicine, vol. 51, no. 2, pp. 257-281, 2011.

[24] J. Wang, H. Feng, J. Zhang, and H. Jiang, "Lithium and valproate acid protect NSC34 cells from $\mathrm{H}_{2} \mathrm{O}_{2}$-induced oxidative stress and upregulate expressions of SIRT3 and CARM1," Neuroendocrinology Letters, vol. 34, no. 7, pp. 648-654, 2013.

[25] Z. Wu, T. W. Lauer, A. Sick, S. F. Hackett, and P. A. Campochiaro, "Oxidative stress modulates complement factor $\mathrm{H}$ expression in retinal pigmented epithelial cells by acetylation of $\mathrm{FOXO}_{3}$," The Journal of Biological Chemistry, vol. 282, no. 31, pp. 22414-22425, 2007.

[26] S. Bhattacharya, E. Chaum, D. A. Johnson, and L. R. Johnson, "Age-related susceptibility to apoptosis in human retinal pigment epithelial cells is triggered by disruption of p53-Mdm2 association," Investigative Ophthalmology \& Visual Science, vol. 53, no. 13, pp. 8350-8366, 2012.

[27] A. Sharma, R. Sharma, P. Chaudhary et al., "4-hydroxynonenal induces p53-mediated apoptosis in retinal pigment epithelial cells," Archives of Biochemistry and Biophysics, vol. 480, no. 2, pp. 85-94, 2008.

[28] M. Pesonen, M. Pasanen, J. Loikkanen et al., "Chloropicrin induces endoplasmic reticulum stress in human retinal pigment epithelial cells," Toxicology Letters, vol. 211, no. 3, pp. 239-245, 2012.

[29] H. Vaziri, S. K. Dessain, E. N. Eaton et al., " $h S I R 2^{\text {SIRT1 }}$ functions as an NAD-dependent p53 deacetylase," Cell, vol. 107, no. 2, pp. 149-159, 2001.

[30] F. Scalera, B. Fulge, J. Martens-Lobenhoffer, A. Heimburg, and S. M. Bode-Böger, "Red wine decreases asymmetric dimethylarginine via SIRT1 induction in human endothelial cells," Biochemical and Biophysical Research Communications, vol. 390, no. 3, pp. 703-709, 2009.

[31] V. Calvanese, E. Lara, B. Suárez-Álvarez et al., "Sirtuin 1 regulation of developmental genes during differentiation of stem cells," Proceedings of the National Academy of Sciences of the United States of America, vol. 107, no. 31, pp. 13736-13741, 2010.

[32] L. Pang, H. Tian, N. Chang et al., "Loss of CARM1 is linked to reduced HuR function in replicative senescence," BMC Molecular Biology, vol. 14, article 15, 2013.

[33] F. Giacco and M. Brownlee, "Oxidative stress and diabetic complications," Circulation Research, vol. 107, no. 9, pp. 10581070, 2010.

[34] D.-I. Kim, M.-J. Park, S.-K. Lim et al., "High-glucose-induced CARM1 expression regulates apoptosis of human retinal pigment epithelial cells via histone 3 arginine 17 dimethylation: role in diabetic retinopathy," Archives of Biochemistry and Biophysics, vol. 560, pp. 36-43, 2014. 


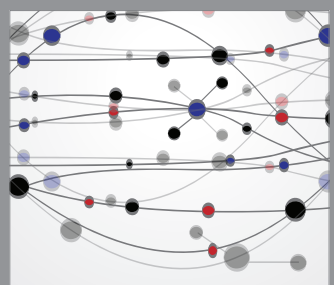

The Scientific World Journal
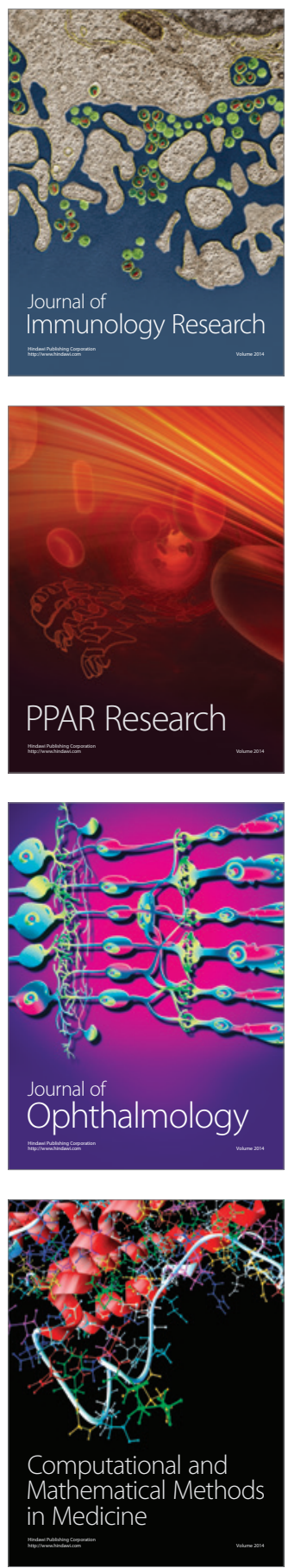

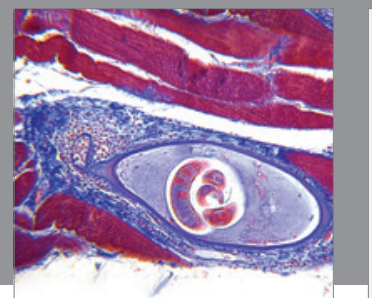

Gastroenterology

Research and Practice
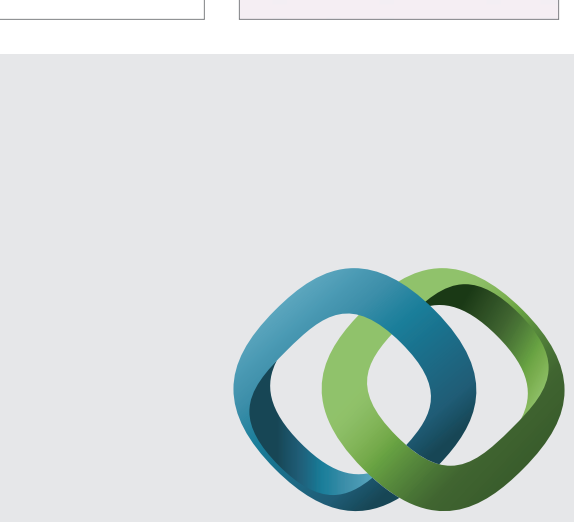

\section{Hindawi}

Submit your manuscripts at

http://www.hindawi.com
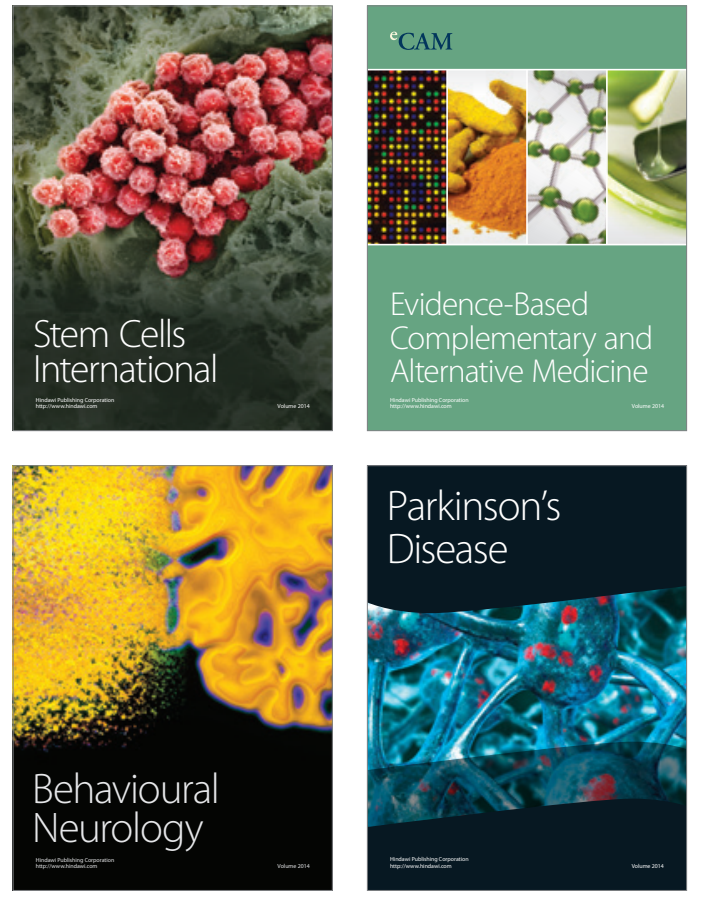
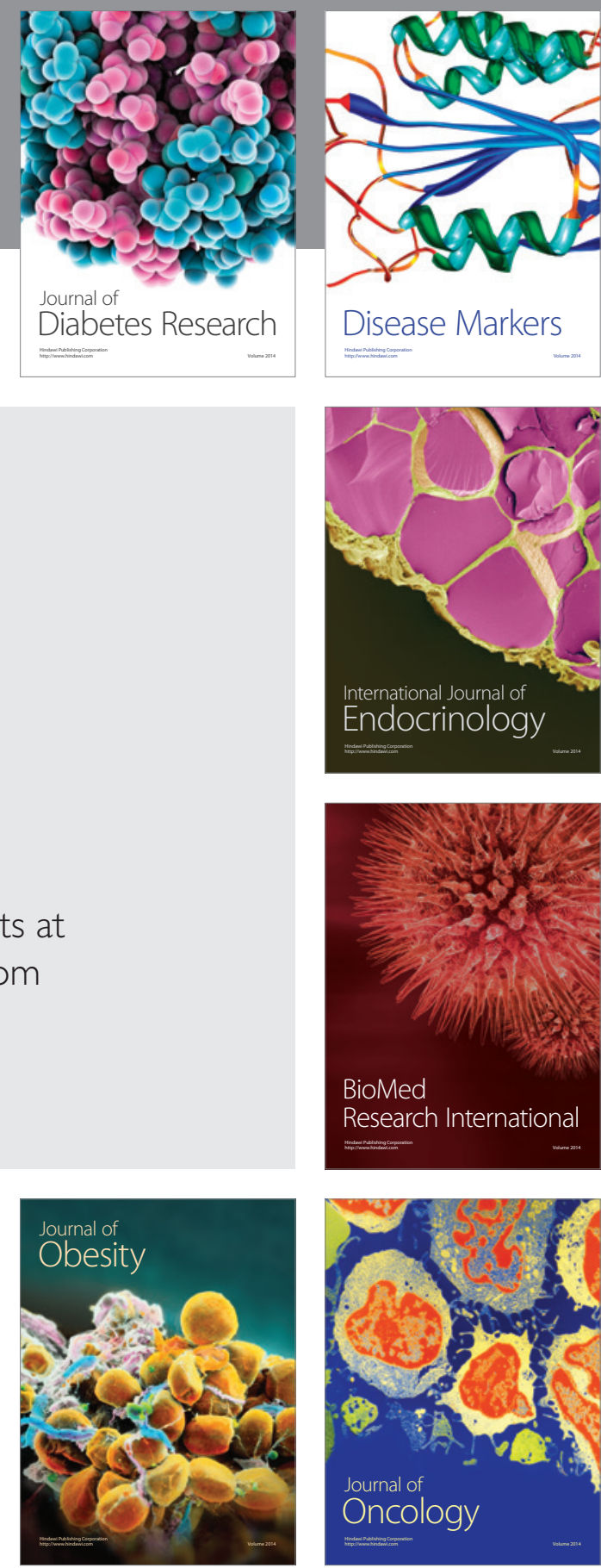

Disease Markers
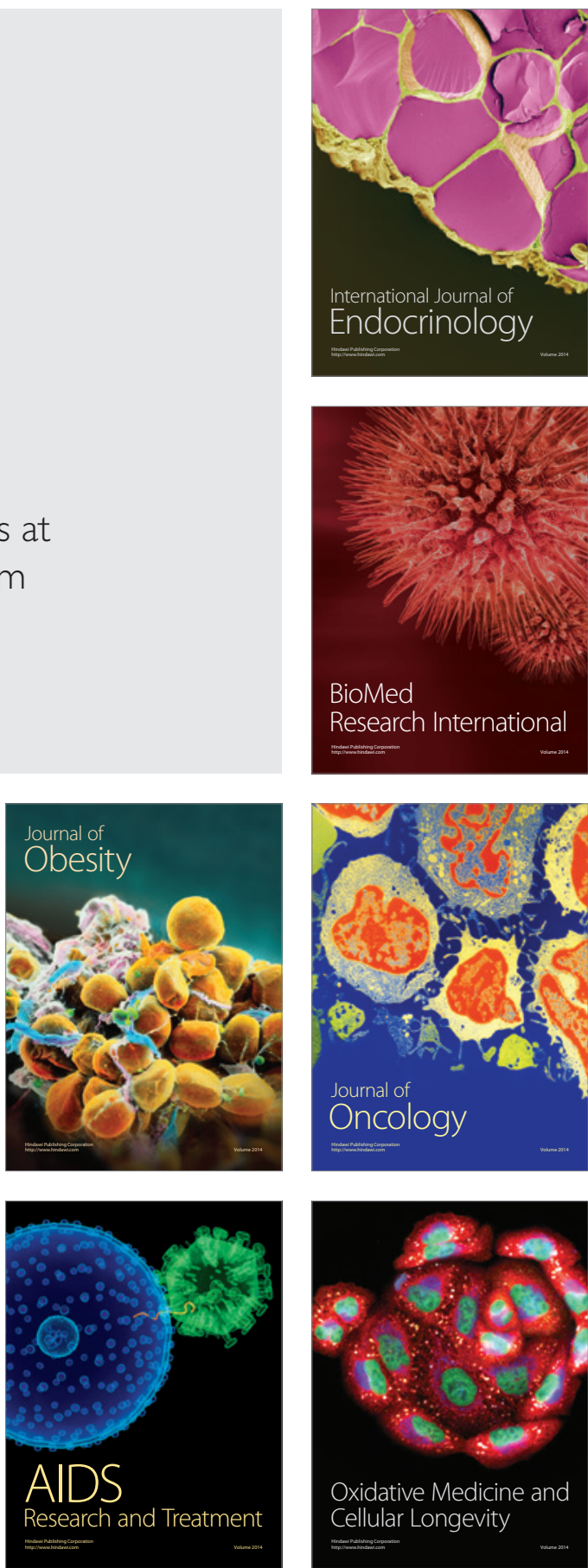